\title{
IN-PLANE PROPAGATING COUPLED PHOTON-PHONON-MAGNETOPLASMONS, THEIR FIELD STRUCTURE AND DISPERSION IN GaAs/AlAs SUPERLATTICES
}

\author{
R. Brazis, R. Narkowicz \\ Semiconductor Physics Institute, A. Goštanto 11, 2600 Vilnius, Lithuania \\ and Polish University at the Association of Polish Scientists of Lithuania \\ P.O. Box 823, 2055 Vilnius, Lithuania
}

AND L. SAFonova

Semiconductor Physics Institute, A. Goštauto 11, 2600 Vilnius, Lithuania

The dispersion and field structure of in-plane propagating $p$-polarized waves in $n$-GaAs/AlAs superlattice is studied in the frequency range below $10 \mathrm{THz}$ in the presence of the steady magnetic field which is perpendicular both to the direction of propagation and periodicity. The existence of non-localized photon-phonon-plasmon modes as well as the magnetic-field-controlled mode localization at selected set of interfaces is predicted.

PACS numbers: 78.66.-w

\section{Introduction}

In-plane propagating modes in superlattices (SL) are of interest for material science because they give information on the anisotropic properties hardly accessible in other ways $[1,2]$. In the effective medium approximation (EMA), the component of SL dielectric tensor along the uniaxis has the poles not coinciding with that of the host materials, and it is not determinable from the normal-incidence data [3]. On the other hand, the localized modes being out of the EMA limits are of special interest because of their sensitivity to the properties of interfaces. Magnetic field is expected to control the mode frequency and localization. Therefore, the theory of coupled mode dispersion and field structure accounting for the influence of steady magnetic fields needs to be developed presenting the aim of this paper. 


\section{Problem formulation}

The SL is formed by alternating layers of two materials with the frequency-dependent dielectric functions $\varepsilon_{1}(\omega)$ and $\varepsilon_{2}(\omega)$. The coupled excitations in particular layers satisfy the Maxwell equations with the boundary conditions of continuity and periodicity at the interfaces. We shall confine our investigation to the case of $p$-polarized wave, supposing that the steady magnetic field oriented along the $z$-axis is perpendicular both to the wave vector $k$ ( $y$-axis) and to the periodicity direction ( $x$-axis). The dispersion relation is

$$
\cos \alpha_{1} d_{1} \cos \alpha_{2} d_{2}-\frac{\varepsilon_{2} \varepsilon_{2}}{2 \alpha_{1} \alpha_{2}}\left(\frac{\alpha_{2}^{2}}{\varepsilon_{2}}+\frac{\beta^{2}}{\varepsilon_{1} \varepsilon_{x x}}\right) \sin \alpha_{1} d_{1} \sin \alpha_{2} d_{2}=1
$$

where $\alpha_{1,2}=\left(k_{0}^{2} \varepsilon_{1,2}-k^{2}\right)^{1 / 2}$ are the amplitude transverse decay constants, $\beta=$ $\left(k_{0}^{2} \varepsilon_{x x}-k^{2}\right)^{1 / 2}, \varepsilon_{1}=\left(\varepsilon_{x x}^{2}-\varepsilon_{x y}^{2}\right) / \varepsilon_{x x}$ is the effective dielectric function of the doped material ( $n$-type GaAs),

$$
\varepsilon_{x x}=\varepsilon_{\mathrm{L} 1}\left(\frac{\omega_{\mathrm{L} 1}^{2}-\omega^{2}}{\omega_{\mathrm{T} 1}^{2}-\omega^{2}}-\frac{\omega_{\mathrm{p}}^{2}}{\omega^{2}-\omega_{\mathrm{c}}^{2}}\right), \quad \varepsilon_{x y}=-\mathrm{i} \varepsilon_{\mathrm{L}} \frac{\omega_{\mathrm{p}}^{2} \omega_{\mathrm{c}}}{\omega\left(\omega^{2}-\omega_{\mathrm{c}}^{2}\right)},
$$

$\varepsilon_{2}=\varepsilon_{\mathrm{L} 2}\left(\omega_{\mathrm{L} 2}^{2}-\omega^{2}\right) /\left(\omega_{\mathrm{T} 2}^{2}-\omega\right)$ is the scalar dielectric function of the undoped material (AlAs), $\varepsilon_{\mathrm{L} 1}$ and $\varepsilon_{\mathrm{L} 2}$ are the high-frequency background dielectric constants, $d=d_{1}+d_{2}$ is the spatial period of SL, $\omega_{\mathrm{T}}$ and $\omega_{\mathrm{L}}$ are the frequencies of transverse and longitudinal optical phonons, $\omega_{\mathrm{p}}$ and $\omega_{\mathrm{c}}$ are the plasma and the cyclotron frequencies, and $k_{0}=\omega / c$.

With these assumptions, the dispersion relation (1) is solved numerically and the values of $\omega$ and $k$ are used to calculate the field amplitude distribution according to [4]. The doped layers are supposed to be thick compared to the undoped ones: $d_{1}=2.25 \mu \mathrm{m}(\mathrm{GaAs})$ and $d_{2}=0.25 \mu \mathrm{m}$ (AlAs). The carrier density in GaAs layers $n=6 \times 10^{16} \mathrm{~cm}^{-3}$.

\section{Results and discussion}

The coupled excitation spectrum exhibits numerous dispersion branches with the lowest three being the most influenced by plasmons. These are labelled with letter $P$ (Fig. 1). The letters $a-d$ mark the values of $\omega$ and $k$ used for the field distribution calculations. The upper frequency region, in the vicinity of the AlAs Reststrahlen band, is not shown and will be discussed elsewhere.

The position of a low-group-velocity region in the middle part of the branch $P 1$ (Fig. 1A) agrees with the characteristic frequency of non-localized modes $\omega_{\mathrm{b}}^{-}$, obtained analytically in EMA neglecting the phonon effects [2]. The influence of phonons in the region shown in Fig. 1A results mainly in slight shift of the second branch $(P 2)$ cut-off frequency with respect to the value $\omega_{0}^{-}$. The latter can be obtained in EMA including plasmon-photon coupling only [2].

Dispersion branches below the Reststrahlen region of GaAs (Fig. 1B) are almost identical to those of bulk $\mathrm{GaAs}$ and their characteristic frequencies agree well with the values of $\omega_{\mathrm{R}}$ and $\omega_{0}$, obtained under the conditions of $\varepsilon_{1} \rightarrow \infty$ and $\varepsilon_{1}=0$. The SL effects are rather negligible here except the branch $P 3$ which is 

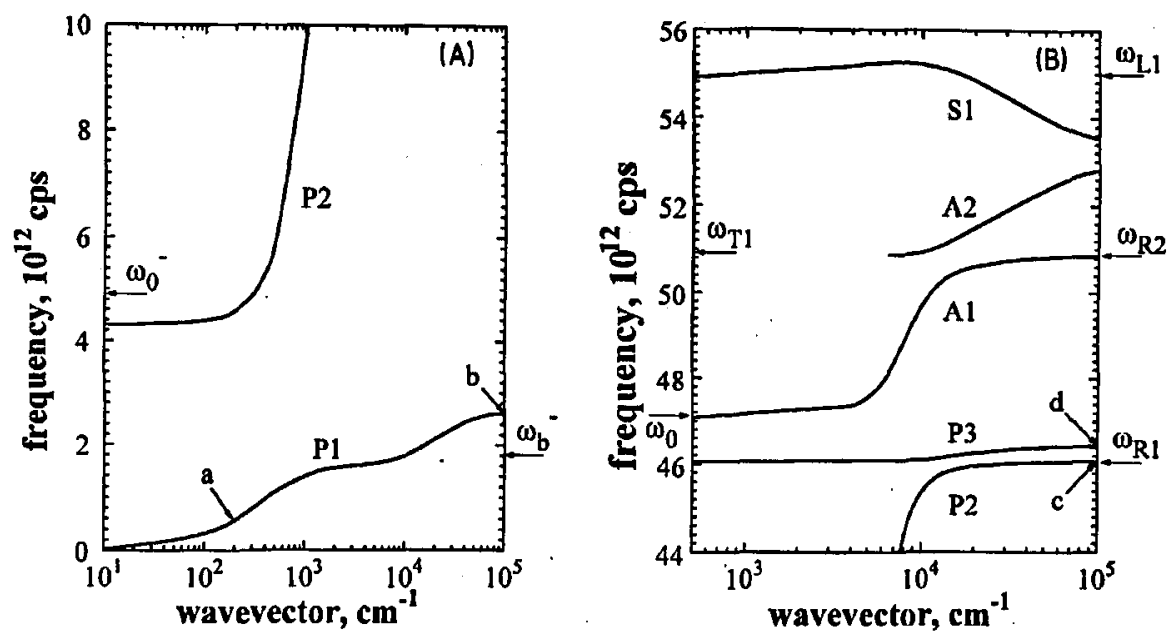

Fig. 1. The dispersion branches of $n$-GaAs/AlAs superlattice in dc magnetic field $B=17 \mathrm{~T}$.

typical of semiconductor-insulator SL [4] with characteristic frequencies shifted by the phonon influence. Inside the Reststrahlen region of $\mathrm{GaAs}$ the most pronounced effect of magnetoplasmons is a maximum of dispersion branch $S 1$ situated above $\omega_{\mathbf{L} 1}$ and related to the non-localized modes. This feature was absent in the spectra studied previously [5].
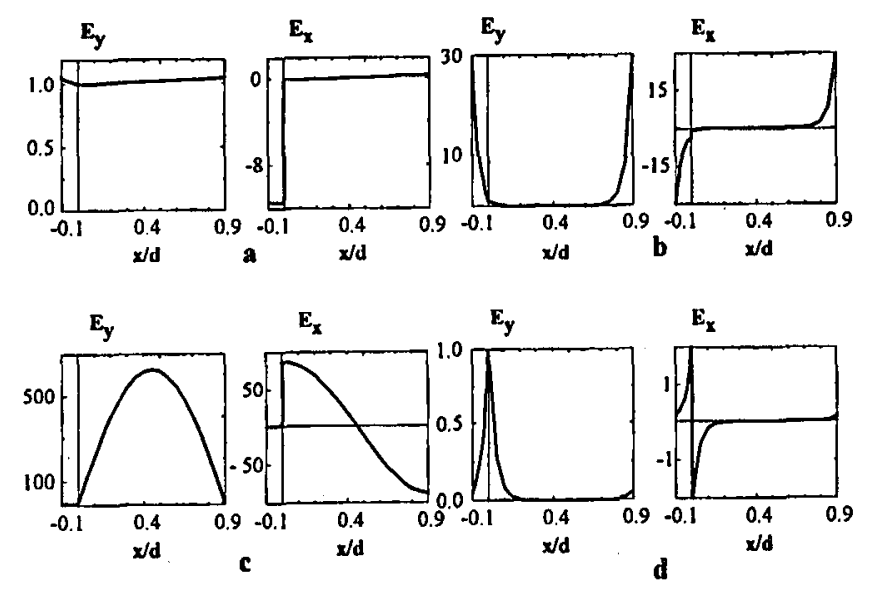

Fig. 2. Field structure in the unit cell of $n$-GaAs/AlAs superlattice corresponding to the points $a-d$ in Fig. 1.

The field distribution for the branch $P 1$ at small values of $k$ (Fig. 2a) presents the slab-guided modes. With the rise of $k$ the field distribution becomes nearly uniform across the spatial period showing the formation of a non-localized mode 
$\left(\omega=\omega_{b}^{-}\right)$which is not encountered in the propagation across the layers. At very large values of $k$ the interface modes are observed (Fig. 2b). Their fields are seen to decay from the unit cell boundaries, not from each interface. Magnetic field- or wave vector inversion shifts the mode localization to the other set of interfaces. This non-reciprocity is not encountered in the absence of magnetic field.

The second dispersion branch $(P 2)$ has a typical of non-localized modes distribution which transforms into the mode perfectly confined within doped layers with the rise of $k$ (Fig. 2c). Dispersion branch $P 3$ at high values of $k$ represents an interface mode (Fig. 2d) shifted by the value $d_{2}$ with respect to the analogous mode discussed above.

Branch $A 1$ at small values of $k$ is characterized by the longitudinal component of electric field nearly constant over the unit cell, whereas the transverse one is a step-like function being nearly zero in undoped layers. This distribution has no analogy in SL studied earlier. With the rise of $k$ the confined mode forms similar to that in undoped SL [5].

The coupled phonon-plasmon-photon mode dispersion is quite easily controlled by changing free carrier concentration (by temperature, photo-, or electric-field-induced ionization), or by dc magnetic fields. The latter has an additional effect of localizing the interface excitations at selected sets of interfaces. This is important for the infrared testing of interfaces in SL, as well as for non-reciprocal optoelectronic devices.

\section{References}

[1] B. Samson, T. Dumelow, A.A. Hamilton, T.J. Parker, S.R.P. Smith, D.R. Tilley, C.T. Foxon, D. Hilton, K. Moore, Phys. Rev. B 46, 2375 (1992).

[2] R.S. Brazis, L.S. Safonova, Int. J. Infrared Millim. Waves 8, 449 (1987).

[3] B. Lou, Solid State Commun. 76, 1395 (1990).

[4] R.S. Brazis, L.S. Safonova, Fiz. Tekh. Poluprovodn. 22, 320 (1988).

[5] R. Brazis, L. Safonova, R. Narkowicz, Infrared Phys. Technol. 36, 51 (1995). 